\title{
Autopsy-Proven Intravascular Lymphoma Presenting as Rapidly Recurrent Strokes
}

\author{
Daisuke Usuda ${ }^{a, b}$ Masahisa Arahata ${ }^{c}$ Rie Temaru ${ }^{c}$ Yoshitsugu Iinuma $^{a}$ \\ Tsugiyasu Kanda $^{b}$ Shinichi Hayashi ${ }^{d}$ \\ ${ }^{a}$ Department of Infectious Diseases, Kanazawa Medical University, Uchinada, ${ }^{b}$ Department \\ of Community Medicine, Kanazawa Medical University Himi Municipal Hospital, Himi, \\ ${ }^{c}$ Department of Internal Medicine, Nanto Municipal Hospital, Nanto, and ${ }^{\mathrm{d}}$ Department of \\ Diagnostic Pathology, Toyama University Hospital, Toyama, Japan
}

\section{Key Words}

Intravascular lymphoma · Rapidly recurrent strokes · Autopsy

\begin{abstract}
We present a 79-year-old Japanese woman diagnosed with cerebral infarction. In spite of enough antiplatelet and anticoagulant therapy, she presented rapidly recurrent strokes three times for 3 months. Magnetic resonance imaging showed progression of bilateral cerebral infarcts, and chest-abdominal computed tomography showed multiple bilateral nodular lesions in the lung and multiple tumor lesions in the liver. Autopsy revealed diagnosis of intravascular lymphoma (IVL). This case indicates that IVL is rare and usually goes undiagnosed until time of autopsy because of its protean neurological manifestations; hence, it should be considered as a possible etiology if multiple strokes occur in a short period of time.
\end{abstract}

\section{Introduction}

Intravascular large B-cell lymphoma (IVL) is a rare entity individualized in the WHO classification since 2001 as a subtype of extranodal diffuse large B-cell lymphoma, which is characterized by the selective growth of lymphoma cells within the lumina of small- and medium-sized vessels, without the involvement of adjacent parenchymal tissue [1-3]. Pathogenesis of IVL remains inadequately understood [4]. Primarily it affects the middle-aged to

\section{KARGER}

Daisuke Usuda

Department of Infectious Diseases, Kanazawa Medical University

1-1 Daigaku, Uchinada-machi, Kahoku-gun, Ishikawa-ken 920-0293 (Japan)

E-Mail united19771108@yahoo.co.jp 
Usuda et al.: Autopsy-Proven Intravascular Lymphoma Presenting as Rapidly Recurrent Strokes

elderly population with a poor performance status, with a slight predominance in men, with an estimated incidence of $<1$ case per million people [5]. Overall mortality rate is thought to be $>80 \%$, and $>50 \%$ of patients are diagnosed at postmortem examination [5]. According to some paper, of patients with IVL, 88\% were diagnosed with B-cell lymphoma, $6 \%$ with T-cell lymphoma, and $2 \%$ with NK cell lymphoma [3]. It is difficult to distinguish between IVL and lymphomas originating from extranodular organs with systemic dissemination into extranodular organs [6].

From a literature review, we did not find any case of IVL with unexplainable multiple recurrent strokes in a short period of time, three times for 3 months, in Japan. We herein report a case with unexplainable multiple recurrent strokes for whom the diagnosis was made at autopsy.

\section{Case Report}

A 79-year-old woman was admitted to our hospital with cognitive decline. Her family history included death of both of her parents caused by cerebral infarction. Her medical history included an operation to appendicitis, type 2 diabetes mellitus and dyslipidemia; meanwhile, it did not include thrombosis. Her vital signs were within normal limits in the examination room. She did not have symptoms of respiratory or gastrointestinal infection or receive any vaccination in the past 2 months. A physical examination showed muscle weakness in the right side of her body. Routine blood tests showed anemia, hypoalbuminemia, elevation of C-reactive protein, alkaline phosphatase, lactate dehydrogenase (LDH), $\gamma$ glutamyltransferase, phosphorus, blood sugar, and hemoglobin A1c, and decrease of calcium and total cholesterol; meanwhile, it did not show coagulation disorder. An electrocardiogram showed normal sinus rhythm without atrial fibrillation, and a carotid ultrasonography showed mild arteriosclerosis. The brain magnetic resonance imaging (MRI) showed multiple ischemic lesions including in the left temporal-parietal region and bilateral cerebellum which were hyperintense on T2-weighted imaging (T2WI; fig. 1).

Considering her age and past history including type 2 diabetes mellitus and dyslipidemia, she was treated with aspirin $200 \mathrm{mg}$ and ticlopidine $100 \mathrm{mg}$, together with rehabilitation for suspected ischemic lacunar stroke. Subsequently, sudden onset of unconsciousness (Japan Coma Scale 1), gait disturbance, and incomplete paralysis on the right side of her body was found on hospital day 44. MRI T2WI showed a new ischemic lesion in the left anterior region (fig. 1). Magnetic resonance angiography revealed mild stenosis of the bilateral internal carotid arteries, middle cerebral arteries, and anterior cerebral arteries. She was added anticoagulant therapy by argatroban, heparin, and warfarin for suspected recurrent ischemic stroke. Chest-abdominal computed tomography (CT) showed multiple bilateral nodular lesions in the lung and multiple tumor lesions in the liver; however, her general condition was so poor that we could not perform further minute investigation. Subsequently, sudden onset of consciousness disorder (Japan Coma Scale 3) and incomplete paralysis on the left side of her body was found on hospital day 79, and MRI T2WI on hospital day 94 showed new ischemic lesions in the right anterior region of the cerebrum (fig. 1). Subsequently, we found suspected ischemic lesions expanded by CT scan and her general condition gradually deteriorated; however, we could not understand their cause. Additionally, we found values of biliary enzymes being gradually elevated; hence, we suspected the occurrence of cholecystitis by abdominal CT and administered antibiotics. However, her general condition gradually deteriorated and she died of multiple organ failure on hospital day 123. 
Usuda et al.: Autopsy-Proven Intravascular Lymphoma Presenting as Rapidly Recurrent Strokes

Autopsy revealed two important findings. First, tumor embolism caused by atypical lymphoid cells was confirmed in vessels of brain, lung, and liver, namely dissemination to solid organs (fig. 2a, b). Second, tumors in the vessels were found to be B-cell lymphoma according to the morphology and immunophenotype (CD20+; fig. 2c). On the other hand, we did not confirm neoplastic proliferation into bone marrow. We suspected diagnosis of lymphoma and measured the value of soluble interleukin-2 receptor using plasma taken at hospital day 44 , and it was remarkably high $(3,300 \mathrm{U} / \mathrm{ml})$. In conclusion, pathological diagnosis was revealed as tumor embolism caused by intravascular B-cell lymphoma, while we clinically diagnosed her illness as recurrent cerebral infarction before the autopsy.

\section{Discussion}

In this case, we could not suspect IVL as one of differential diagnosis until the time of autopsy because of nonspecific clinical presentation, laboratory and image findings. The common differential diagnoses of rapidly recurrent multiple stroke-like episodes include embolism of cardiac origin, demyelination diseases such as multiple sclerosis or acute disseminating encephalomyelitis, cerebral venous thrombosis, paraneoplastic syndromes, thyroid disorders, vasculitis, infection, or neoplasm [7]. The present case did not have atrial fibrillation, valvular heart disease, or other cardiac disorders predisposing to cardioembolic stroke, but she had diabetes and dyslipidemia, which were considered as cardiovascular risks. Demyelination diseases were not likely for either patient considering lack of history of recent infection or vaccination, and absence of typical chronic course of disease progression. IVLs showing only neurologic manifestations might be misdiagnosed as cerebral infarctions [8], and IVLs sometimes cause unexplained multifocal and recurrent strokes. Therefore, IVL should be considered as a possible etiology if multiple strokes occur in a short period of time, but it is so difficult [9]. For patients with recurrent stroke-like events despite of antithrombotic therapies, the accurate diagnosis requires a high index of suspicion by the neurologist and a brain biopsy specimen demonstrating lymphoma cells within the lumen of cerebral blood vessels [10].

The initial MRI of the brain showed multifocal plaque-like central nervous system (CNS) lesions suggestive of multiple sclerosis, and follow-up MRI studies suggested new parenchymal brain lesions consistent with multiple evolving embolic strokes and subsequently with brain infarcts [2]. Abnormal findings in brain MRI of IVL patients are as follows: (1) infarct-like lesions, (2) nonspecific white matter lesions, (3) meningeal enhancement, (4) mass-like lesions, and (5) hyperintense lesions in the pons on T2WI [7]. Cerebral MRI findings in patients with IVL are also diverse; these variations make diagnosis challenging and it is difficult to distinguish tumor embolism from thromboembolism [7]. Therefore, in this case, we could not consider MRI findings as tumor embolism. Importantly, neuroimaging discloses CNS involvement only in half of patients with neurological symptoms because there are no pathognomonic neuroradiological findings for IVL; ischemic foci are the most common presentation pattern, and therefore, vasculitis is the most common differential diagnosis [11]. Therefore, we should not deny the possibility of IVL because of absence abnormality in imaging procedure [12]. On the other hand, (18)F-fluorodeoxyglucose (FDG) positron emission tomography/CT imaging showed increased FDG uptake in the involved organs; therefore, it can be used in suspected cases of IVL for early diagnosis of this rapidly fatal malignancy [13].

A systemic evaluation to determine the proper biopsy site is needed for early diagnosis [1]. However, clinical presentations are nonspecific, focal space-occupying lesions or lym- 
Usuda et al.: Autopsy-Proven Intravascular Lymphoma Presenting as Rapidly Recurrent Strokes

phoadenopathy are always lacking, and due to its aggressive behavior, it often leads to a delayed diagnosis, and diagnosis is made postmortem in half of the cases $[2,5]$. Among them, CNS IVL had the highest proportion, $60 \%$ compared to $8 \%$ of skin, $11 \%$ of bone marrow and spleen, and 7\% of lung IVLs [3]. Therefore, organ biopsies are mandatory for accurate IVL diagnosis; however, no standard procedure has been established [4]. On the other hand, minimally invasive and highly sensitive procedures are required for its accurate diagnosis: bone marrow aspiration and biopsy, and random skin biopsy at an early stage are recommended [6], although, in this case, the proliferation of IVL cells was not confirmed in bone marrow. Random skin biopsy from normal-appearing skin is highly sensitive in the diagnosis of IVL, comparable to bone marrow trephine biopsy [14]. It should be performed irrespective of the presence or absence of skin lesions in patients who were suspicious of IVL [14]. Therefore, if we had suspected the existence of lymphoma as one of differential diagnosis when we found tumors over her whole body, we could have performed random skin biopsy and made a correct diagnosis of IVL. She could have undergone skin biopsy even if her general condition was so poor that she could not undergo lung or liver biopsy.

According to the WHO classification, IVL is defined as a lesion being limited inside vessels; therefore, our case cannot strictly satisfy the criteria of IVL. However, tumor formations or invasion to the outside of vessels are often found in advanced cases or autopsy, and lesions or situations are suspected to be developed from IVL; hence, a recent convincing view is that we diagnose IVL when the lesion is found in the small vessel or sinusoid [15]. In conclusion, we herein reported a case report of IVL which presented as recurrent and stepwise strokes in a short time of period. IVL should be considered as a possible etiology if multiple strokes occur in a short period of time.

\section{Statement of Ethics}

We declare that written informed consent for publication was obtained from the patient.

\section{Disclosure Statement}

The authors state that they have no conflict of interest.

\section{References}

1 Kanazawa Y, Hagiwara N, Matsuo R, Arakawa S, Ago T, Kitazono T: Case of intravascular large B-cell lymphoma (IVLBCL) with central nervous system symptoms diagnosed by renal biopsy. Rinsho Shinkeigaku 2014;54:484-488.

2 Dasanu CA, Codreanu I, Mesologites T, Ali S, Sullivan P, Jethava A: Western variant of brain intravascular lymphoma displaying three distinct evolutive radiologic stages. Conn Med 2014;78:73-76.

3 Fonkem E, Lok E, Robison D, Gautam S, Wong ET: The natural history of intravascular lymphomatosis. Cancer Med 2014;3:1010-1024.

-4 Hoshi T, Fujii Y, Okuyama S, Tanaka Y, Kimura N, Mouri Y, Takaya H, Kajimura K: Two cases of intravascular lymphoma diagnosed by random liver biopsy. Nihon Shokakibyo Gakkai Zasshi 2014;111:1433-1440.

5 Wahie S, Dayala S, Husain A, Summerfield G, Hervey V, Langtry JA: Cutaneous features of intravascular lymphoma. Clin Exp Dermatol 2011;36:288-291.

-6 Masaki Y, Miki M, Sakai T, Sawaki T, Fukushima T, Umehara H: Intravascular large B-cell lymphoma: an important cause of fever of unknown origin. Brain Nerve 2011;63:435-441.

7 Yamamoto A, Kikuchi Y, Homma K, O’uchi T, Furui S: Characteristics of intravascular large B-cell lymphoma on cerebral MR imaging. AJNR Am J Neuroradiol 2012;33:292-296. 
Usuda et al.: Autopsy-Proven Intravascular Lymphoma Presenting as Rapidly Recurrent Strokes

8 Momota H, Narita Y, Miyakita Y, Shibui S: Intravascular lymphoma of the central nervous system presenting as multiple cerebral infarctions. Nagoya J Med Sci 2012;74:353-358.

-9 Hung LC, Tsai JH, Wu CS, Dai YC, Chen CC, Sung SF: Brain biopsy-proven intravascular lymphomatosis presenting as rapidly recurrent strokes-two case reports. Acta Neurol Taiwan 2014;23:11-18.

10 Sengupta S, Pedersen NP, Davis JE, Rojas R, Reddy H, Kasper E, Greenstein P, Wong ET: Illusion of stroke: intravascular lymphomatosis. Rev Neurol Dis 2011;8:e107-e113.

11 Mihaljevic B, Sternic N, Skender Gazibara M, Sretenovic A, Antic D, Terzic T, Kostic V: Intravascular large Bcell lymphoma of central nervous system - a report of two cases and literature review. Clin Neuropathol 2010;29:233-238.

12 van Rhenen A, van de Donk NW: Rapidly progressive neurological deterioration without magnetic resonance imaging abnormalities: intravascular lymphoma. Blood 2014;123:2914.

13 Hong JY, Kim HJ, Ko YH, Choi JY, Jung CW, Kim SJ, Kim WS: Clinical features and treatment outcomes of intravascular large B-cell lymphoma: a single-center experience in Korea. Acta Haematol 2014;131:18-27.

14 Matsue K, Asada N, Odawara J, Aoki T, Kimura S, Iwama K, Fujiwara H, Yamakura M, Takeuchi M: Random skin biopsy and bone marrow biopsy for diagnosis of intravascular large B cell lymphoma. Ann Hematol 2011;90:417-421.

15 Ponzoni M, Ferreri AJ, Campo E, Facchetti F, Mazzucchelli L, Yoshino T, Murase T, Pileri SA, Doglioni C, Zucca E, Cavalli F, Nakamura S: Definition, diagnosis, and management of intravascular large B-cell lymphoma: proposals and perspectives from an international consensus meeting. J Clin Oncol 2007;25:3168-3173.

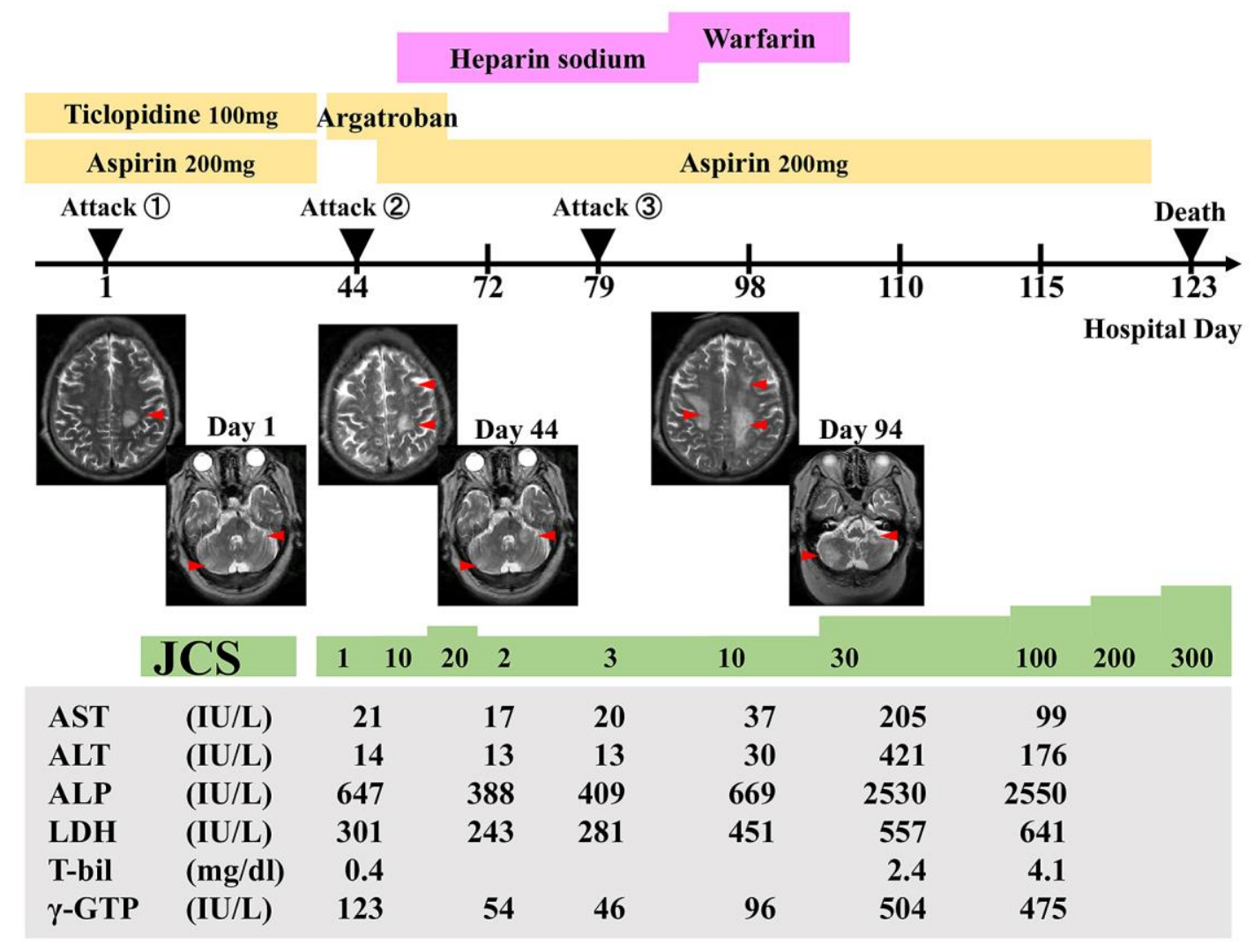

Fig. 1. Clinical course of the patient. In the clinical course, brain attack had been suspected three times by the findings of MRI T2WI on hospital days 1, 44 and 79. The patient deceased on hospital day 123. JCS = Japan Coma Scale. 
Usuda et al.: Autopsy-Proven Intravascular Lymphoma Presenting as Rapidly Recurrent Strokes
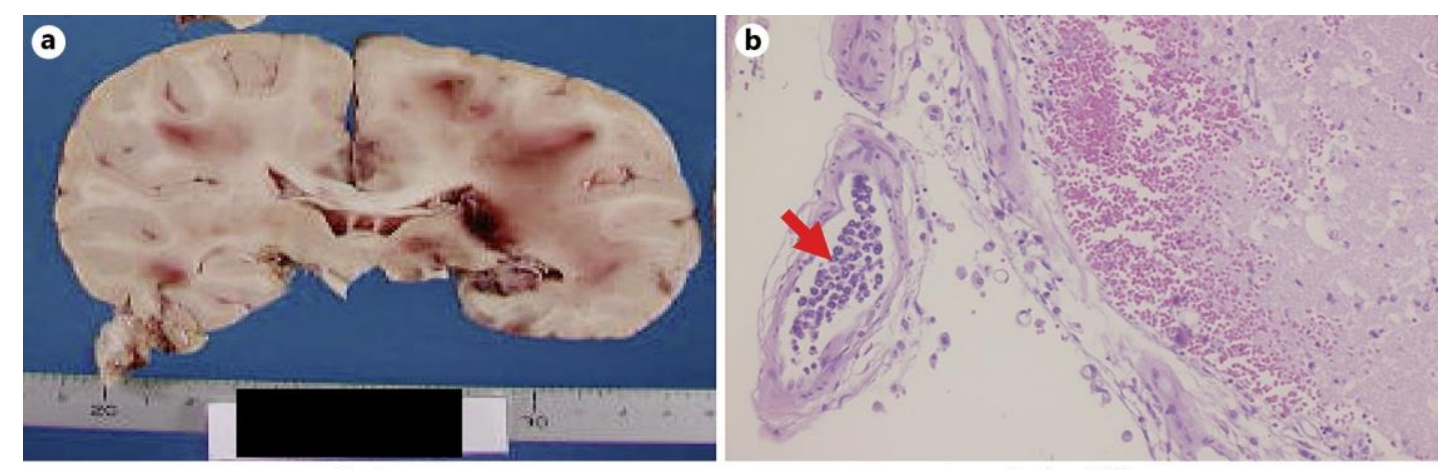

Brain

Brain $\times 200$

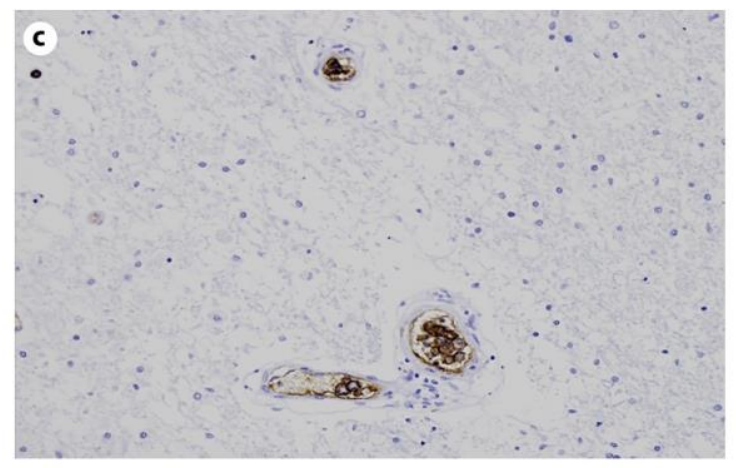

Brain $\times 200$

Fig. 2. Histopathological images of the brain. a Macroscopic view of the specimen. Tumors are confirmed as red color lesion. b Lymphoma cell invasion is confirmed inside the vessel of the vein (red arrow). Hematoxylin and eosin staining $(\times 200)$. c The immunohistochemical study of lymphoma cell is positive for CD20 $(\times 200)$. 